Cite this: Phys. Chem. Chem. Phys., 2014, 16, 7584

Received 9th December 2013, Accepted 17th February 2014

DOI: $10.1039 / \mathrm{c} 3 \mathrm{cp} 55197 \mathrm{k}$

www.rsc.org/pccp

\section{Magnetic field dependent long-lived spin states in amino acids and dipeptides}

\author{
Andrey N. Pravdivtsev, ${ }^{\text {ab }}$ Alexandra V. Yurkovskaya, ${ }^{\text {ab }}$ Herbert Zimmermann, ${ }^{c}$ \\ Hans-Martin Vieth ${ }^{d}$ and Konstantin L. Ivanov*ab
}

\begin{abstract}
Magnetic field dependence of long-lived spin states (LLSs) of the $\beta-\mathrm{CH}_{2}$ protons of aromatic amino acids was studied. LLSs are spin states, which are immune to dipolar relaxation, thus having lifetimes far exceeding the longitudinal relaxation times; the simplest example of an LLS is given by the singlet state of two coupled spins. LLSs were created by means of the photo-chemically induced dynamic nuclear polarization technique. The systems studied were amino acids, histidine and tyrosine, with different isotopomers. For labeled amino acids with the $\alpha-\mathrm{CH}$ and aromatic protons substituted by deuterium at low fields the LLS lifetime, $T_{\text {LLS }}$, for the $\beta-\mathrm{CH}_{2}$ protons was more than 40 times longer than the $T_{1}$-relaxation time. Upon increasing the number of protons the ratio $T_{L L S} / T_{1}$ was reduced; however, even in the fully protonated amino acids it was about 10; that is, the long-lived mode was still preserved in the system. In addition, the effect of paramagnetic impurities on spin relaxation was studied; field dependencies of $T_{1}$ and $T_{\text {LLS }}$ were measured. LLSs were also formed in tyrosine-containing dyads; a $T_{\text {LLS }} / T_{1}$ ratio of $\sim 7$ was found, usable for extending the spin polarization lifetime in such systems.
\end{abstract}

\section{Introduction}

Investigation of relaxation phenomena in NMR spectroscopy is a well-known method to obtain information about molecular mobility in solution (notably, for proteins and bio-molecules), liquid crystals, etc. An interesting special case of nuclear spin relaxation is given by relaxometry of long-lived spin states (LLSs), ${ }^{1-3}$ which have attracted significant attention during the last decade. ${ }^{1-13}$ By an LLS we mean a nuclear spin state having a lifetime, $T_{\text {LLS }}$, much longer than the longitudinal relaxation time, $T_{1}$. LLSs arise from the fact that in a scalar-coupled spin system in a certain magnetic field range (as will be discussed below) some of the eigen-states are the same for the static and fluctuating parts of the spin Hamiltonian. ${ }^{2,3}$ As a consequence, their relaxation rate, $R_{\mathrm{LLS}}=1 / T_{\mathrm{LLS}}$, is considerably slower than the relaxation rate of the net polarization, $R_{1}=1 / T_{1}$. The simplest example of an LLS is the singlet state of a 2 -spin system, ${ }^{1-3}$ which is immune to dipolar relaxation that usually is the main source of relaxation in solutions. The singlet state is an eigen-state of both the static and fluctuating dipolar spin Hamiltonian at low magnetic field, consequently its relaxation rate $\left(R_{\mathrm{LLS}}=1 / T_{\mathrm{LLS}}=0\right)$

\footnotetext{
${ }^{a}$ International Tomography Center SB RAS, Institutskaya 3a, Novosibirsk, 630090, Russia. E-mail: ivanov@tomo.nsc.ru; Fax: +7-383-333-1399; Tel: +7-383-330-8868

${ }^{b}$ Novosibirsk State University, Pirogova 2, Novosibirsk, 630090, Russia

${ }^{c}$ Max-Planck-Institut für Medizinische Forschung, Jahnstrasse 29 D-69120 Heidelberg, Germany

${ }^{d}$ Freie Universität Berlin, Arnimallee 14, Berlin, D-14195, Germany
}

becomes zero. In general, the dipolar relaxation rate of the LLS of two scalar-coupled spins at arbitrary magnetic field $B$ is: ${ }^{5,11}$

$$
R_{\mathrm{LLS}}=\frac{20+2 \cos 4 \theta-\sqrt{2(81-80 \cos 4 \theta+\cos 8 \theta)}}{60} R_{1}
$$

where $R_{1}=\frac{1}{T_{1}}$ is the rate of $T_{1}$-relaxation, $\theta=\frac{1}{2} \arctan \frac{J}{\delta \nu(\mathrm{B})}$ is the 'mixing' angle for the two-spin system; here $J$ is the scalar spin-spin coupling and $\delta \nu(B)=\left(\nu_{1}-\nu_{2}\right)$ is the difference in Larmor precession frequency of the spins at the field $B$. In the present work we will use the following notion of the coupling regime between spins that can be determined by the mixing angle $\theta$ : two spins are strongly coupled when $|\theta| \rightarrow \frac{\pi}{4}$ and weakly coupled when $|\theta| \rightarrow 0$. It follows from eqn (1) that in the strong coupling regime $R_{\mathrm{LLS}} \rightarrow 0$, which leads to a drastic increase of the relaxation time. In the weak coupling regime $R_{\mathrm{LLS}} \rightarrow \frac{R_{1}}{3}$ : thus, $T_{\mathrm{LLS}}$ is still longer than $T_{1}$ but the lifetime increase is not so pronounced as that at low magnetic field. The reduction of $T_{\mathrm{LLS}}$ is caused by the fact that in the weak coupling regime the singlet state is no longer an eigen-state of the static Hamiltonian. Although the dipolar interaction is not the only source of relaxation it is usually the predominant one; thus, at low fields $T_{\text {LLS }}$ can be up to 45 times longer than $T_{1} ;{ }^{5}$ for heteronuclei, namely ${ }^{15} \mathrm{~N}$, the $T_{\mathrm{LLS}}$ times are extremely long reaching impressive values such as 26 min. ${ }^{14}$ Although usually LLSs in two-spin systems are discussed, LLSs exist in the multispin 
system too although the prolongation of the lifetime is usually smaller. ${ }^{5,8,10,15-19}$ In previous investigations it was shown that exploiting an LLS allows one to study molecular geometry in multispin systems, namely, to determine molecular torsion angles. ${ }^{8}$

LLSs can also be exploited to study slow processes; in addition, they are frequently utilized in connection with spin hyperpolarization (HP), which is a promising strategy for increasing the low inherent sensitivity of NMR spectroscopy. There are a few HP techniques like Dynamic Nuclear Polarization (DNP), ${ }^{20-22}$ Para-Hydrogen Induced Polarization (PHIP), ${ }^{23,24}$ Chemically Induced Dynamic Nuclear Polarization (CIDNP), ${ }^{25}$ which have received much attention during the last decade. One of the main problems in HP is increasing its lifetime because it usually decays with $T_{1}$ thus being relatively short-lived. In CIDNP and PHIP experiments long-lived spin order can be formed directly in the polarization process without additional NMR pulses. ${ }^{5,18,26,27}$ HP generated by means of DNP is usually net-polarization, which relaxes with $T_{1}$, but can be transformed into long-lived spin order by application of special RF-pulse sequences. For this reason, new NMR methods for converting the longitudinal spin order into LLSs (and back) have been developed. ${ }^{26,28,29}$ Only in the special case of very strong DNP singlet order can be formed directly and does not require applying additional RF-pulses. ${ }^{30}$ Thus, exploiting LLSs to store $\mathrm{HP}$ of different kind is possible and opens up a prospect for new applications of NMR spectroscopy; in particular, it will allow one to store HP for extended time periods and to investigate ${ }^{31,32}$ slow dynamic processes which are masked by $T_{1}$.

In a recent study ${ }^{5}$ we suggested to use photo-CIDNP for creating LLSs and investigated the magnetic field dependence of $T_{\mathrm{LLS}}$. In the CIDNP method HP is generated as a result of electron-spin selective radical recombination and nuclear-spin dependent intersystem crossing in a transient radical pair. It has been shown earlier ${ }^{5,33-35}$ that using photo-CIDNP at low magnetic field allows one to selectively populate spin states in the diamagnetic reaction product with respect to their total spin quantum number. For a two-spin system this results in an overpopulated or an underpopulated nuclear singlet state as compared with the triplet states. The resulting spin order is long-lived with a lifetime equal to $T_{\mathrm{LLS}}$. Therefore photo-CIDNP at low magnetic field allows one to increase the lifetime of HP by a factor of $T_{\mathrm{LLS}} / T_{1}$ along with increasing the NMR signal for particular lines by a factor of about 100 (related to the Boltzmann polarization at magnetic field of 7 Tesla). ${ }^{5}$ This was demonstrated for the $\beta-\mathrm{CH}_{2}$ protons of histidine. These protons have a $T_{1}$ of about 1 second while $T_{\mathrm{LLS}}$ is about 40-60 seconds in partially deuterated histidine. The $\beta-\mathrm{CH}_{2}$ protons are important in the context of LLSs because they are present in most of the natural amino acids and have a rather short $T_{1}$ that is usually less than 1 second. Therefore increasing their relaxation time in combination with the NMR enhancement provided by HP is promising. In ref. 5 the whole field dependence of $T_{\mathrm{LLS}}$ (relaxation dispersion) was measured for protonated and deuterated histidine. However, the field dependence $T_{\mathrm{LLS}}$ of the $\beta-\mathrm{CH}_{2}$ protons of histidine was not analyzed quantitatively, nor was a sharp feature observed in the $T_{\text {LLS }}$ field dependence of fully protonated histidine modeled.
In the present work we continue the study of $T_{\mathrm{LLS}}$ in amino acids, namely, we investigate histidine and tyrosine amino acids in aqueous solution with deuteration at different positions. It allows us to investigate the influence of neighboring nuclei on the lifetime of LLSs in the field range from $0.1 \mathrm{mT}$ up to $7 \mathrm{~T}$. We will show that a continuous increase of the number of protons leads to a decrease of $T_{\text {LLS }}$. The appearance of sharp features in the field dependencies of $T_{\mathrm{LLS}}$ at specific field positions can be explained as an effect of nuclear spin Level Anti-Crossings (LACs). We will also study the influence of paramagnetic additions on $T_{\mathrm{LLS}}$, a topic that is important, e.g., for DNP-related applications of LLSs. Finally, we will study the field dependence of $T_{\text {LLS }}$ in Tyr-containing dyads. They have a rather short $T_{1}$, which can be a severe limitation of HP studies in such systems. Using LLSs in this case is thus crucial for preserving spin polarization sufficiently long. All results will be modeled quantitatively using a density matrix approach and the Redfield theory of spin relaxation.

Our study is thus relevant for using the LLS methodology to create and preserve HP in biological molecules in aqueous solutions and in biological tissues. In particular, we will measure the full field dependence of $T_{\mathrm{LLS}}$, since this is of importance to understand the nature of LLSs and to optimize their 'longevity', i.e., the $T_{\mathrm{LLS}} / T_{1}$ ratio. Another focus of our work is considering systems of more than two spins, since such systems are commonly met. Moreover, in comparison with two-spin systems they have additional characteristic features in their relaxation behavior, for instance, those coming from LACs.

\section{Materials and methods}

\section{A. Field-cycling}

All experiments were carried out using a $7 \mathrm{~T}(300 \mathrm{MHz}) \mathrm{NMR}$ spectrometer equipped with a fast field-cycling device. ${ }^{36}$ The field-cycling method is based on mechanical shuttling of the NMR probehead in the fringe magnetic field along the bore axis of the spectrometer cryo-magnet. Changing the location of the probehead enables setting the magnetic field in a range from $50 \mathrm{mT}$ up to $7 \mathrm{~T}$. Magnetic fields below $50 \mathrm{mT}$ are adjusted by an additional system of electromagnets. The minimal time of shuttling the probehead from $0.1 \mathrm{mT}$ (the lowest position) up to the detection field of $B_{0}=7 \mathrm{~T}$ is $\sim 0.3 \mathrm{~s}$. Using slow sample rotation and a zero-susceptibility material for the field-cycling probehead allows us to detect ${ }^{1} \mathrm{H}$ NMR spectra with a spectral resolution of about $0.3 \mathrm{~Hz}$.

A pulsed excimer XeCl laser with a wavelength $\lambda=308 \mathrm{~nm}$ and a repetition rate of $100 \mathrm{~Hz}$ was used for initiating the photoreactions, which led to the CIDNP formation. The probehead is equipped with a flexible light guide, thus allowing us to initiate photo-CIDNP at arbitrary magnetic fields under constant irradiation conditions.

\section{B. Experimental protocol}

Our previous results ${ }^{5}$ demonstrate that by photo-CIDNP at low magnetic field LLSs can be formed. In the present investigation we used the same way of creating LLSs in all systems under study. 


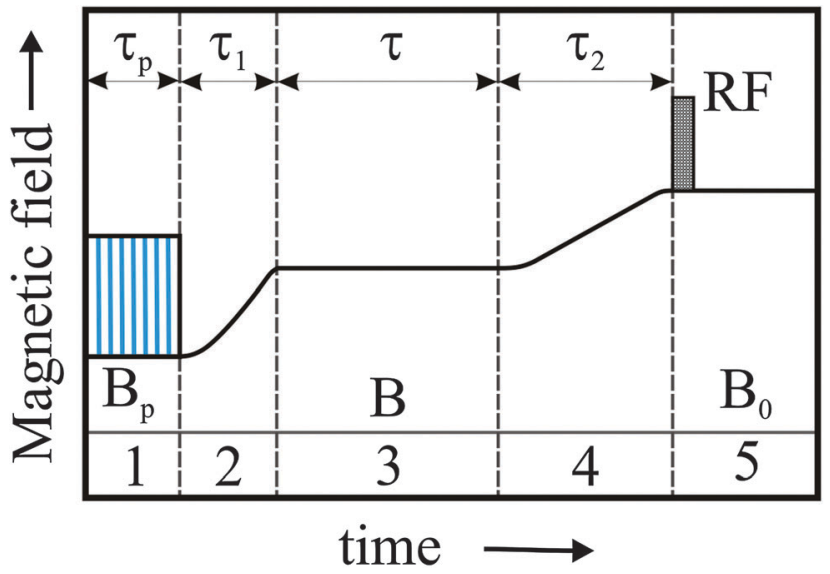

Fig. 1 Experimental protocol for studying LLSs at variable magnetic field using photo-CIDNP. (1) During the time period $\tau_{\mathrm{p}}$ the sample is irradiated by a laser at the field $B_{\mathrm{p}}$. At this stage photo-CIDNP is formed. (2) External magnetic field is changed from $B_{\mathrm{p}}$ to $B$ during the time period $\tau_{1}$. (3) CIDNP generated at stage (1) relaxes to the thermal equilibrium polarization at the field $B$ during the time period $\tau$. (4) The external magnetic field changes from the relaxation field $B$ to the detection field $B_{0}$ during time $\tau_{2}$. (5) An RF pulse is applied and the free induction decay is observed at $B_{0}=7 \mathrm{~T}$.

The experimental protocol for measuring the lifetime of hyperpolarization is shown in Fig. 1. The protocol consists of 5 consecutive stages. At the first stage (preparation) the sample is irradiated by the laser during the time period $\tau_{\mathrm{p}}$ at the field $B_{\mathrm{p}}$; during this stage the required HP is generated. At the second stage (field variation 1 ) the probe with the sample is shuttled during the time $\tau_{1}$ to the variable intermediate field $B$. At the third stage (relaxation) the spin system relaxes to the equilibrium polarization at this field during the time $\tau$. At the fourth stage (field variation 2) the probehead with the sample is shuttled during the time period $\tau_{2}$ to the observation field $B_{0}$ of the NMR spectrometer. Finally, at the fifth stage (detection) a $45^{\circ}$ or $90^{\circ}$ RF-pulse is applied and the Free Induction Decay (FID) is recorded. After Fourier transformation of the FID, individual lines in the proton multiplets of high-resolution NMR spectra are analyzed. The time traces of the line intensities were simulated by the sum of two exponential functions. The longest decrement was attributed to $T_{\mathrm{LLS}}$ at the field $B$. In our experiments the setting of field $B_{\mathrm{p}}$ was $0.1 \mathrm{mT}$ when $B>30 \mathrm{mT}$ and $B_{\mathrm{p}}=B$ when $B<30 \mathrm{mT}$. The experiment was carried out in this way because the fields below $30 \mathrm{mT}$ are adjusted by the system of electromagnets with high field-homogeneity (less than $10 \mathrm{mT} \mathrm{m}^{-1}$ ), which is, however, not designed for fast field switching. Therefore to avoid the slow stage of adjusting the magnetic field by switching the electric current and in order to keep the high field-homogeneity for $B<30 \mathrm{mT}$ we have taken $B_{\mathrm{p}}=B$, thus sacrificing the optimum HP; accordingly, the second stage in the protocol was omitted.

\section{Simulation method}

For simulating the experiment we used the same theory as before. ${ }^{4,35,37}$ It takes into account every stage of the experimental protocol. For modelling the generation of HP by means of photo-CIDNP we used a Green function approach ${ }^{38,39}$ for calculating the spin evolution in radical pairs. ${ }^{35,37}$ It allowed us to calculate the starting populations of the spin states of the diamagnetic reaction product. These state populations were used as the initial conditions for modelling the subsequent stages in the experimental protocol. For the following three stages the Liouville-von Neumann equation for the spin density matrix was numerically solved as explained in our previous studies. $^{40,41}$ For modelling the relaxation the intramolecular dipolar interactions were calculated from the actual molecular geometry. For calculating the relaxation operator we assumed that the molecule was tumbling as a whole; the correlation times, $\tau_{\mathrm{c}}, 100 \mathrm{ps}$ for amino acids and $200 \mathrm{ps}$ for dipeptides and dyads were used. In the whole range of magnetic fields under investigation the product of $\tau_{\mathrm{c}}$ and the Larmor precession frequency of proton is much smaller than $1\left(\omega \tau_{\mathrm{c}} \ll 1\right)$ therefore it was possible to use the extreme narrowing limit ${ }^{42}$ for calculating the relaxation operator. Also the relaxation super-operator was simplified. We considered only relaxation of the eigen-state populations, whereas relaxation of coherences was completely neglected; the elements of the relaxation matrix were calculated in the same way as before. ${ }^{4,18,19}$ After modeling the first four stages we calculated the kinetics of net polarization for all spins in the system as a function of $B$ and $\tau,\left\langle\hat{I}_{z}^{k}\right\rangle(\tau, B)$, and for the $\beta-\mathrm{CH}_{2}$ protons we calculated in addition the evolution of the following spin order $\left\langle\hat{I}_{z}^{\beta 1}-\hat{I}_{z}^{\beta 2}\right\rangle(\tau, B)$ (for the reason explained below). After that the time traces were simulated by a mono-exponential decay for the net-polarization, $\left\langle\hat{I}_{z}^{k}\right\rangle$, the decrement was attributed to $T_{1}$, and by a bi-exponential decay for the $\left\langle\hat{I}_{z}^{\beta 1}-\hat{I}_{z}^{\beta 2}\right\rangle$ term, with the longest decrement defined as $T_{\mathrm{LLS}}$. Also the field dependence of the $T_{1}$-relaxation times was calculated with the aim of checking the validity of our approach. As has been found earlier, the calculation method described gives accurate predictions not only for the high-field $T_{1}$-relaxation times but also for the whole $T_{1}(B)$ dependence. ${ }^{4}$

As was discussed before the long-lived singlet spin state in the two-spin system of the $\beta-\mathrm{CH}_{2}$ protons corresponds to the difference in populations of the singlet and triplet states, which is given by the spin order $\left(\hat{\mathbf{I}}^{\beta 1} \cdot \hat{\mathbf{I}}^{\beta 2}\right)$. Previously it was shown ${ }^{4,43}$ that in the case of adiabatic field cycling such spin order at low magnetic field is correlated with the $\left(\hat{I}_{z}^{\beta 1}-\hat{I}_{z}^{\beta 2}\right)$ spin order at high magnetic field. As far as the $\beta-\mathrm{CH}_{2}$ protons are concerned the conditions of adiabaticity are always fulfilled, as follows from our numerical calculations. In fact, for the $\beta-\mathrm{CH}_{2}$ protons adiabaticity is provided by the large spin-spin interaction (the largest in the molecules studied) and the small chemical shift difference (about $0.15 \mathrm{ppm}$ ). The lifetime of the singlet state thus can be determined by analyzing the term $\left\langle\hat{I}_{z}^{\beta 1}-\hat{I}_{z}^{\beta 2}\right\rangle$ as a function of $\tau$. Interestingly, for the $\beta-\mathrm{CH}_{2}$ protons in the amino acids under study CIDNP results in overpopulated triplet states and underpopulated singlet states; ${ }^{35,37}$ i.e., the polarized molecules are formed predominantly not in the singlet state but in the triplet state. Nonetheless, the resulting spin order (given by the difference in the singlet and triplet state populations) is long-lived and relaxes with the $T_{\text {LLS }}$ time, which does not depend on the preparation method. 
When in the case of a two-spin system only dipolar relaxation is considered the approach described above gives infinite $T_{\text {LLS }}$ in contrast to experimental observations. This is because other mechanisms of relaxation are present that relax the singlet state. To take into account such sources of relaxation we added a field-independent constant $R_{0}$ to the relaxation rates of both net polarization, $R_{1}$, and long-lived singlet spin order, $R_{\mathrm{LLS}}$; the $R_{0}$ value was determined from fitting of the experimental data. This was done for coupled two-spin systems, since for higher-spin systems the time $T_{\mathrm{LLS}}$ is always finite. The only exception of a scalar coupled multi-spin system where it was found necessary to take $R_{0} \neq 0$ was L-tyrosine. However, in all cases other relaxation sources contribute only slightly to the relaxation rates: the rate $R_{0}$ was always small as compared to the high-field relaxation rates: $R_{0} \ll R_{1}\left(B_{0}\right)$. Thus, in our treatment we either neglected other relaxation mechanisms (such as the chemical shift anisotropy, which is small for protons) or took them into account phenomenologically as additional small fieldindependent contributions.

All NMR parameters used (chemical shifts, $J$-couplings, hyperfine interaction (HFI) constants, $T_{1}$-relaxation times at high field) for all protons of amino acids and dyads under

Table $1 \mathrm{~J}$-couplings, chemical shifts, $T_{1}$-relaxation times at $7 \mathrm{~T}$ for $\mathrm{His}$ and $\mathrm{N}$-AC-His and HFI constants of His* $(g=2.00226)^{44}$

\begin{tabular}{llllll}
\hline$J / \mathrm{Hz}$ & $\beta 1$ & $\beta 2$ & $\alpha$ & $\mathrm{H} 2$ & $\mathrm{H} 5$ \\
\hline$\beta 1$ & & - & - & - & - \\
$\beta 2$ & -14.8 & & - & - & - \\
$\alpha$ & 4.9 & 8.2 & & - & - \\
$\mathrm{H} 2$ & - & - & - & & - \\
$\mathrm{H} 5$ & -0.8 & -0.5 & - & 1.6 & \\
$\delta / \mathrm{ppm}$ & 2.96 & 2.81 & 4.356 & 7.65 & 7.0 \\
$T_{1}(7 \mathrm{~T}) / \mathrm{s}$ & 0.75 & 0.75 & 4.4 & 24.4 & 11.1 \\
$T_{1}(7 \mathrm{~T}) / \mathrm{s}\left(\right.$ purified from $\left.\mathrm{O}_{2}\right)$ & 1.25 & 1.24 & - & - & - \\
HFI (His ${ }^{\bullet} / \mathrm{mT}$ & 1.53 & 1.53 & -0.03 & -1.21 & -1.06
\end{tabular}

investigation are shown in Tables 1 and 2. Chemical shifts and $J$-couplings were determined by simulation of the NMR spectra taken at 7 T. HFI constants and $g$-factors of the radicals have been determined before. ${ }^{44} T_{1}$-relaxation times were measured at $7 \mathrm{~T}$ using the conventional inversion-recovery scheme and were used for comparison with the results of the calculation described above and with the $T_{\text {LLS }}$ times. Distances between the protons that were used for calculating dipolar interactions were obtained from a quantum chemical calculation performed in ORCA $^{45}$ using a B3LYP functional and a cc-pVDZ basis set in aqueous solution. Distances used for calculating the dipolar relaxation operator are shown in Tables 3 and 4 .

\section{Chemical compounds}

The dye 2,2'-dipyridyl (DP), the solvent $\mathrm{D}_{2} \mathrm{O}$, as well as $\mathrm{DCl}$ and $\mathrm{NaOD}$ that were used to adjust the proper $\mathrm{pH}$ were obtained from Sigma-Aldrich. The amino acids $N$-acetyl-Lhistidine (N-Ac-His), L-histidine (His), N-acetyl-L-tyrosine (N-Ac-Tyr), and L-tyrosine (Tyr) were obtained from Bachem. The dipeptides $N$-acetyl tyrosyl-tyrosine ( $N$-Ac-Tyr-Tyr) and tryptophyl-tyrosine (Trp-Tyr) were obtained from Sigma-Aldrich. The dyad 4-carboxybenzophenone-Tyrosine (4CBP-Tyr) ${ }^{46,47}$ was kindly provided by Dr G. Hörner; the nitroxyl radical 4-hydroxyTEMPO (TEMPOL) was synthesized by us according to the

Table 3 Distances between the protons in N-Ac-His taken for calculation of the dipolar relaxation operator

\begin{tabular}{llllll}
\hline$r / \AA$ & $\beta 1$ & $\beta 2$ & $\alpha$ & H2 & H5 \\
\hline$\beta 1$ & & - & - & - & - \\
$\beta 2$ & 1.802 & & - & - & - \\
$\alpha$ & 2.548 & 3.061 & & - & - \\
$\mathrm{H} 2$ & 5.251 & 5.164 & 4.724 & & - \\
$\mathrm{H} 5$ & 2.784 & 3.137 & 4.668 & 4.255 &
\end{tabular}

Table $2 \mathrm{~J}$-couplings, chemical shifts, $T_{1}$-relaxation times at $7 \mathrm{~T}$ for Tyr and tyrosine containing derivatives and $\mathrm{HFI}$ constants of Tyr $(g=2.0045)^{44}$

\begin{tabular}{|c|c|c|c|c|c|c|c|}
\hline$J / \mathrm{Hz}$ & $\beta 1$ & $\beta 2$ & $\alpha$ & $\mathrm{H} 2$ & H6 & H3 & H5 \\
\hline$\beta 2$ & -14.7 & & - & - & - & - & - \\
\hline$\alpha$ & 5.16 & 7.80 & & - & - & - & - \\
\hline H6 & - & - & - & 2.43 & & - & - \\
\hline H3 & - & - & - & 8.36 & 0.27 & & - \\
\hline H5 & - & - & - & 0.41 & 7.44 & 3.14 & \\
\hline$\delta(N$-Ac-Tyr $) / \mathrm{ppm}$ & 3.149 & 2.938 & 4.545 & 7.181 & 7.181 & 6.874 & 6.874 \\
\hline$\delta(N-\mathrm{Ac}-\mathrm{Tyr}-\mathrm{Tyr}) / \mathrm{ppm}$ & 2.84 & 2.63 & 4.6 & 7.02 & 7.02 & 6.82 & 6.82 \\
\hline$\delta($ Trp-Tyr $) /$ ppm & 2.93 & 2.76 & 4.42 & 7.02 & 7.02 & 6.82 & 6.82 \\
\hline$\delta(4 \mathrm{CBP}-\mathrm{Tyr}) / \mathrm{ppm}$ & 3.71 & 3.54 & 5.34 & 7.63 & 7.63 & 7.27 & 7.27 \\
\hline$T_{1}(7 \mathrm{~T}, \mathrm{Tyr}) / \mathrm{s}$ & 0.6 & 0.6 & 1.9 & 1.65 & 1.65 & 2.87 & 2.87 \\
\hline$T_{1}(7 \mathrm{~T}, N-\mathrm{Ac}-\mathrm{Tyr}) / \mathrm{s}$ & 0.62 & 0.62 & 2.05 & 1.74 & 1.74 & 3.2 & 3.2 \\
\hline
\end{tabular}


Table 4 Distances between the protons in N-Ac-Tyr taken for calculation of the dipolar relaxation operator

\begin{tabular}{llllllll}
\hline$r / \AA$ & $\beta 1$ & $\beta 2$ & $\alpha$ & H2 & H6 & H3 & H5 \\
\hline$\beta 1$ & & - & - & - & - & - & - \\
$\beta 2$ & 1.767 & & - & - & - & - & - \\
$\alpha$ & 2.488 & 3.062 & & - & - & - & - \\
H2 & 2.537 & 3.741 & 2.894 & & - & - & - \\
H6 & 3.687 & 2.455 & 4.218 & 4.300 & & - & - \\
H3 & 5.483 & 4.743 & 5.786 & 4.964 & 2.476 & & - \\
H5 & 4.799 & 5.529 & 4.910 & 2.490 & 4.964 & 4.296 & \\
\hline
\end{tabular}

procedure described earlier. ${ }^{48}$ The selectively deuterium substituted amino acids (Tyr- $\mathrm{D}_{2,6}$, Tyr- $\mathrm{D}_{3,5, \alpha}$, Tyr- $\mathrm{D}_{2,3,5,6, \alpha}$, His- $\mathrm{D}_{2, \alpha}$, His- $\mathrm{D}_{2,5, \alpha}$ ) were synthesized according to the protocol described in ref. 49 and 50 using $\mathrm{H} / \mathrm{D}$ exchange under acidic conditions at elevated temperature. Exchange reactions above $100{ }^{\circ} \mathrm{C}$ were carried out in a sealed glass tube. The acid catalyzed exchange reactions were neutralized after the exchange with $\mathrm{NaOD}-\mathrm{D}_{2} \mathrm{O}$ or $\mathrm{NaOH}-\mathrm{H}_{2} \mathrm{O}$ to $\mathrm{pH} 7$.

Three kinds of aqueous solutions were used: (i) $4 \mathrm{mM}$ Tyr or other tyrosine derivatives ( $N$-Ac-Tyr, $N$-Ac-Tyr-Tyr, Trp-Tyr) and $1 \mathrm{mM}$ DP at pH 3.3, (ii) $25 \mathrm{mM}$ His or $N$-Ac-His with $1 \mathrm{mM}$ DP at pH 5, (iii) $4 \mathrm{mM} 4 \mathrm{CBP}-\mathrm{Tyr}$ at $\mathrm{pH} 3.3$ were dissolved in a $1: 1$ mixture of $\mathrm{D}_{2} \mathrm{O}$ and acetonitrile- $\mathrm{D}_{3}$. DP and 4CBP absorb light at the wavelength $\lambda=308 \mathrm{~nm}$ of our laser; the DP concentration was chosen to give an optical density of 3 . For 4 CBP we were using even higher optical density to minimize problems coming from depletion of this dye; then only a small fraction of the 4CBP molecules was excited by each laser pulse, though at the expense of non-uniform sample illumination. The radical pair formation and the pathway of the radical pair evolution for these amino acids and dyes have been investigated before. ${ }^{37,51,52}$ Before each series of experiments every sample after adjusting the $\mathrm{pH}$ was purified from dissolved oxygen by purging it with nitrogen gas for 10 minutes. In the solution of His and DP additional purification presumably from dissolved oxygen by laser irradiation was found; this kind of 'purification' leads to a significant increase of $T_{\mathrm{LLS}}$ (see Section III.A).

Three kinds of dyads were used: N-Ac-Tyr-Tyr, Trp-Tyr, and 4CBP-Tyr. CIDNP formation is different in these dyads: in Trp-Tyr only the tyrosine part is polarized by means of CIDNP. ${ }^{53}$ In $N$-Ac-Tyr-Tyr both ends are hyperpolarized; due to different chemical shifts of the $\beta-\mathrm{CH}_{2}$ protons in the two residues (caused by the presence of the acetyl at the N-terminus) it was possible to measure $T_{\mathrm{LLS}}$ of the $\beta-\mathrm{CH}_{2}$ protons on both ends of the dyad separately. In 4CBP-Tyr photo-CIDNP was formed without an additional dye molecule; the photochemistry of the 4CBP-Tyr dyad has been investigated before. ${ }^{46,47}$

Our investigations show that the stable radical TEMPOL reacts with free radicals of Tyr and DP, which are produced by light irradiation. As a result diamagnetic molecules of 2,2,6,6tetramethyl piperidine-1,4-diol are formed. A similar reaction with TEMPOL was reported before. ${ }^{54}$ To avoid experimental errors coming from such side reactions in experiments involving TEMPOL (see Section III.C) we have taken care that the depletion of the nitroxide was always below $20 \%$.

\section{Results and discussion}

\section{A. LLSs in histidine}

To demonstrate the validity of our experimental and theoretical approach for studying the $T_{\mathrm{LLS}}$ field dependencies in multi-spin systems let us start the discussion from the two-spin system of the $\beta-\mathrm{CH}_{2}$ protons of the amino acid histidine. We will gradually increase the complexity of the system under investigation to the three-spin system of His- $\mathrm{D}_{2, \alpha}$ and finally up to five spins in fully protonated $N$-Ac-His. The $T_{\mathrm{LLS}}$ field dependencies of the $\beta-\mathrm{CH}_{2}$ protons in the histidine case are shown in Fig. 2.

The $T_{\mathrm{LLS}}$ field dependencies for the two-spin system of His- $\mathrm{D}_{2,5, \alpha}$ are in full agreement with the theoretical prediction when the LLS relaxation rate is given by the sum of $R_{\mathrm{LLS}}$ (see eqn (1)) and small $R_{0}$. The $T_{\mathrm{LLS}}$ behaviour is conditioned by the coupling regime of the two protons. As is predicted by the theory, $T_{\mathrm{LLS}}$ is maximal at strong coupling (being equal to $1 / R_{0}$ at zero-field) and decreases with increasing magnetic field (see Fig. 2). Our experiments reveal that both $T_{\mathrm{LLS}}$ and $T_{1}$ gradually increase upon light irradiation (see Fig. 2 and Table 1) and reach their maximal values after about 1000 laser pulses. We suggest that this increase of the relaxation times is due to depletion of dissolved triplet oxygen. Therefore two field dependencies of $T_{\text {LLS }}$ were measured: one with less than 200 pulses and the other with more than 1000 pulses. The LLS lifetime at low magnetic field increases from $30 \mathrm{~s}$ (below 200 laser pulses) up to $60 \mathrm{~s}$ (above 1000 pulses) and $T_{1}$ at $7 \mathrm{~T}$ increases as well from $0.7 \mathrm{~s}$ up to $1.2 \mathrm{~s}$. Hence, in both cases $T_{\mathrm{LLS}}$ is about 43 times longer than $T_{1}: T_{\mathrm{LLS}}(B=0) / T_{1}=1 /\left(R_{0} T_{1}\right) \approx 43$. For other deuteriumsubstitutes of His we did not look in detail at possible 'purification' effects because at zero-field the $T_{\text {LLS }}$ time was considerably shorter $(\approx 16 \mathrm{~s}$ as compared to $\approx 30 \mathrm{~s}$ with the consequence that it becomes more difficult to measure quantitatively the corresponding changes in the relaxation rate).

Increasing the number of proton spins to three in His- $\mathrm{D}_{2, \alpha}$ we observed a similar behaviour of $T_{\mathrm{LLS}}$ (Fig. 2): $T_{\mathrm{LLS}}$ decreases

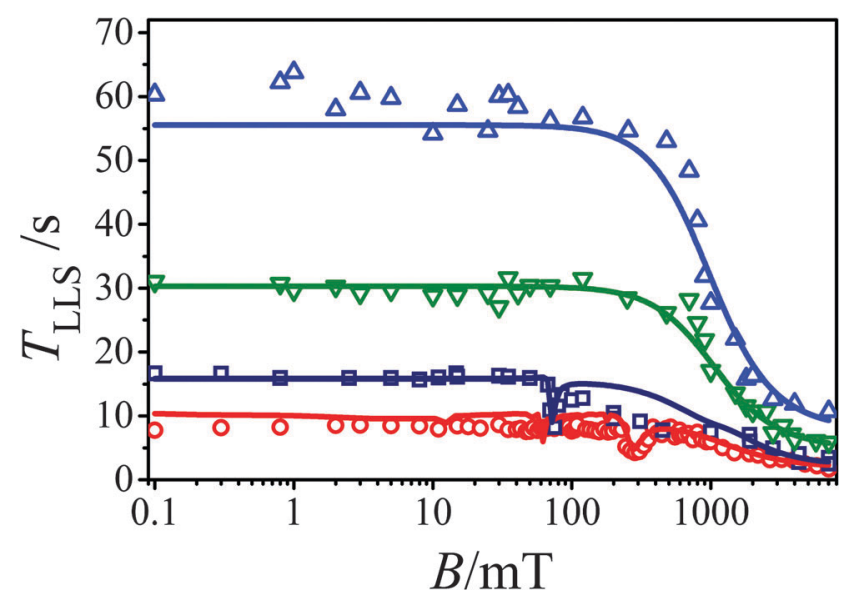

Fig. 2 Field dependencies of $T_{\text {LLS }}$ of the $\beta-\mathrm{CH}_{2}$ protons of $\mathrm{N}-\mathrm{AC}-\mathrm{His}$ (circles), His- $D_{2, \alpha}$ (squares), His- $D_{2,5, \alpha}$ (down triangles), and His- $D_{2,5, \alpha}$ after light irradiation by 1000 pulses (up triangles). Solid lines show the calculation results. 
with increasing field. Although formally the singlet state is not an eigen-state of the spin Hamiltonian, still $T_{\mathrm{LLS}} \gg T_{1}$ and the $T_{\text {LLS }}$ field dependence are similar to that in the two-spin system. This is because of two factors: First, the singlet state is nearly an eigen-state of the static part of the Hamiltonian because of the ' $J$-stabilization' mechanism ${ }^{16}$ reported earlier: the coupling between the $\beta-\mathrm{CH}_{2}$ protons is so large that the singlet state is almost completely 'isolated' from other states, namely, $\left|J_{\beta 1}, \beta_{2}\right|=$ $14.8 \mathrm{~Hz} \gg 0.3 \mathrm{~Hz}=\left|J_{\beta 1, \mathrm{H} 5}-J_{\beta 2}, \mathrm{H} 5\right|$. Second, the singlet state is almost an eigen-state of the stochastic part of the Hamiltonian because of a similar reason. The dipolar interaction between the $\beta-\mathrm{CH}_{2}$ protons is much stronger than those between each of the $\beta-\mathrm{CH}_{2}$ protons and the $\mathrm{H} 5$ proton because the dipolar interaction decreases as $\frac{1}{r^{3}}$ with the distance between the spins, with the distance between the $\beta-\mathrm{CH}_{2}$ protons being the shortest. Hence, long-lived character of the $\beta-\mathrm{CH}_{2}$ proton state survives in His- $\mathrm{D}_{2, \alpha}$, although $T_{\text {LLS }}$ is shorter than in His- $\mathrm{D}_{2,5, \alpha}$.

In the case of the three-spin system of His- $\mathrm{D}_{2, \alpha}$ a new sharp feature appears at around $70 \mathrm{mT}$. The origin of the feature is the scalar coupling between the two $\beta-\mathrm{CH}_{2}$ protons and the $\mathrm{H} 5$ proton (see Chart 1 and Table 1) giving rise to an LAC in the three-spin system. An LAC occurs when upon field variation two energy levels with the same projection of the total nuclear spin, $I_{z}$, tend to cross, but a coupling matrix element between them leads to a splitting of the two energy levels so that the crossing is avoided. This behaviour is termed an LAC or an avoided crossing. In a two-spin system an LAC is always present at zero magnetic fields, while in scalar-coupled multi-spin systems LACs also appear at non-zero fields. In our case the sharp feature at $70 \mathrm{mT}$ appears as a result of an LAC between two states that correlate with the pair $|\beta \alpha \alpha\rangle$ and $|\alpha \alpha \beta\rangle$ of the high-field states (here, as usual, $|\alpha\rangle$ and $|\beta\rangle$ are the spin-up and spin-down Zeeman states);
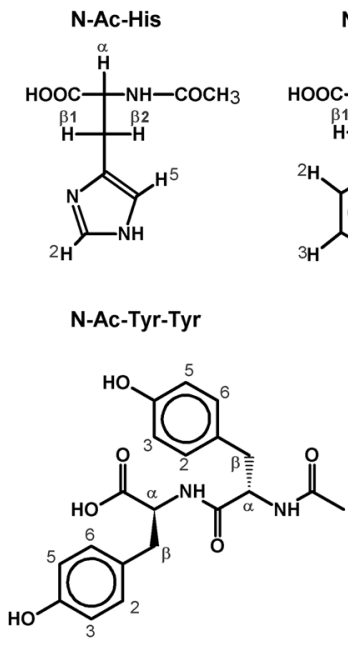

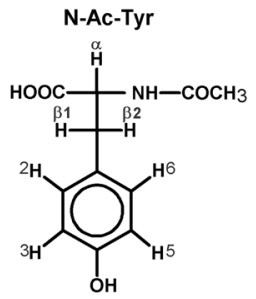

Trp-Tyr
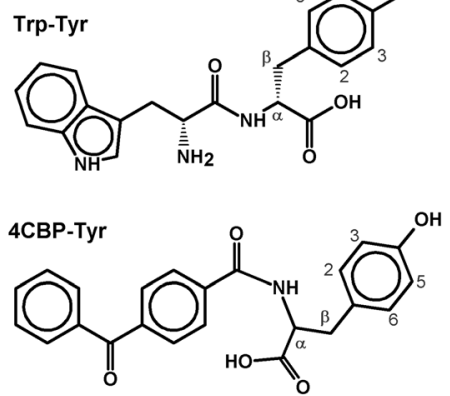

Chart 1 Molecular structure and the numbering scheme of amino acids: $\mathrm{N}$-acetyl-histidine ( $\mathrm{N}$-Ac-His), N-acetyl-tyrosine ( $\mathrm{N}$-Ac-Tyr); dipeptides: $N$-acetyl tyrosyl-tyrosine ( $N$-Ac-Tyr-Tyr), tryptophyl-tyrosine (Trp-Tyr), dyad 4CBP-Tyr, and nitroxyl radical TEMPOL. Numbering of protons is done here according to the IUPAC nomenclature. in the product states the spins are listed in the order $\beta 1-\mathrm{CH}_{2}$, $\beta 2-\mathrm{CH}_{2}, \mathrm{H} 5$. The decrease of $T_{\mathrm{LLS}}$ at the LAC point is caused by mixing the LLS with another state having faster relaxation. As is seen from Fig. 2 our theoretical approach is in good agreement with the experimental observation reproducing the influence of the LAC. At low fields $T_{\mathrm{LLS}}$ is about $16 \mathrm{~s}$ that is 21 times longer than $T_{1}$ at high field. It is worth noting that although the influence of the $\mathrm{H} 5$ proton on the relaxation dispersion of the $\beta-\mathrm{CH}_{2}$ protons is relatively weak, it cannot be described by simply assuming that the $\mathrm{H} 5$ proton only changes the $R_{0}$ value. This is because the scalar couplings with the third proton become important leading to effects, which cannot be taken into account by redefining $R_{0}$. A striking manifestation of the $J$-coupling is the LAC effect seen as a sharp peak in the $T_{\mathrm{LLS}}(B)$ dependence.

Finally, we investigated the $T_{\text {LLS }}$ field dependence in the fivespin system of fully protonated $N$-Ac-His. $T_{\text {LLS }}$ in the five-spin system is the shortest. The further decrease of $T_{\mathrm{LLS}}$ is due to two reasons. First, the main reason is that $J$-stabilization is less efficient here than in His- $\mathrm{D}_{2, \alpha}$ because the scalar couplings between the $\beta-\mathrm{CH}_{2}$ and the $\alpha-\mathrm{CH}$ protons are much bigger than those between the $\beta-\mathrm{CH}_{2}$ and $\mathrm{H} 5$ protons while the difference $\left|J_{\beta 1, \alpha}-J_{\beta 2, \alpha}\right|=3.3 \mathrm{~Hz}$ is only 4.5 times smaller than $\left|J_{\beta 1, \beta 2}\right|$; therefore the corresponding LLS has smaller singlet character. Second, the dipolar interactions between the $\beta-\mathrm{CH}_{2}$ and $\alpha-\mathrm{CH}$ protons are stronger than those between the $\beta-\mathrm{CH}_{2}$ and $\mathrm{H} 5$ protons; this also supports faster relaxation of the LLS. Nevertheless, $T_{\mathrm{LLS}}$ is still 10 times longer than $T_{1}$ at high field. Interestingly, the sharp feature in the $T_{\mathrm{LLS}}$ field dependence is shifted from $70 \mathrm{mT}$ to $300 \mathrm{mT}$. This is explained by an LAC between states that correlate with the high-field states $|\beta \alpha \alpha\rangle$ and $|\alpha \alpha \beta\rangle$ where the nuclei are listed in the following order: $\beta 1-\mathrm{CH}_{2}$, $\beta 2-\mathrm{CH}_{2}, \alpha-\mathrm{CH}$. This LAC occurs at $300 \mathrm{mT}$ as has been found before; ${ }^{40,55}$ often the $\beta-\mathrm{CH}_{2}$ and $\alpha-\mathrm{CH}$ protons of amino acids (namely, in histidine, tryptophan, etc.) have an LAC in this field range. As the relaxation in protonated His is much faster than in deuterated species additional features (e.g., those coming from LACs caused by couplings to the aromatic protons) tend to disappear.

Thus, in all the cases it is seen that our theoretical treatment (solid lines in Fig. 2) perfectly explains not only the main trend of the monotonous increase of $T_{\text {LLS }}$ with decreasing field but also reproduces the sharp features caused by the LACs in scalar coupled multi-spin systems.

\section{B. LLSs in tyrosine}

Having understood the field dependencies of LLSs in histidine let us analyze $T_{\text {LLS }}$ in tyrosine with different isotopomers (see Fig. 3). Here we will start again from the simplest case of the two-spin system of the $\beta-\mathrm{CH}_{2}$ protons of Tyr- $\mathrm{D}_{2,3,5,6, \alpha}$ and Tyr- $\mathrm{D}_{2,6, \alpha}$. Although Tyr- $\mathrm{D}_{2,6, \alpha}$ has four protons $\left(\beta-\mathrm{CH}_{2}, \mathrm{H} 3\right.$ and H5) it is easy to see that the $T_{\mathrm{LLS}}$ field dependencies of Tyr- $\mathrm{D}_{2,3,5,6, \alpha}$ and Tyr- $\mathrm{D}_{2,6, \alpha}$ are almost identical (see Fig. 3) and similar to those of His- $\mathrm{D}_{2,4, \alpha}$ (Fig. 2). It indicates that the scalar coupling and dipolar interaction among the $\mathrm{H} 3, \mathrm{H} 5$ and $\beta-\mathrm{CH}_{2}$ protons, which can lead to a distortion of the singlet state of the $\beta-\mathrm{CH}_{2}$ protons, are negligible. This can be seen from a comparison of the results for the LLS in Tyr- $D_{2,3,5,6, \alpha}$ and Tyr- $D_{2,6, \alpha}$. 

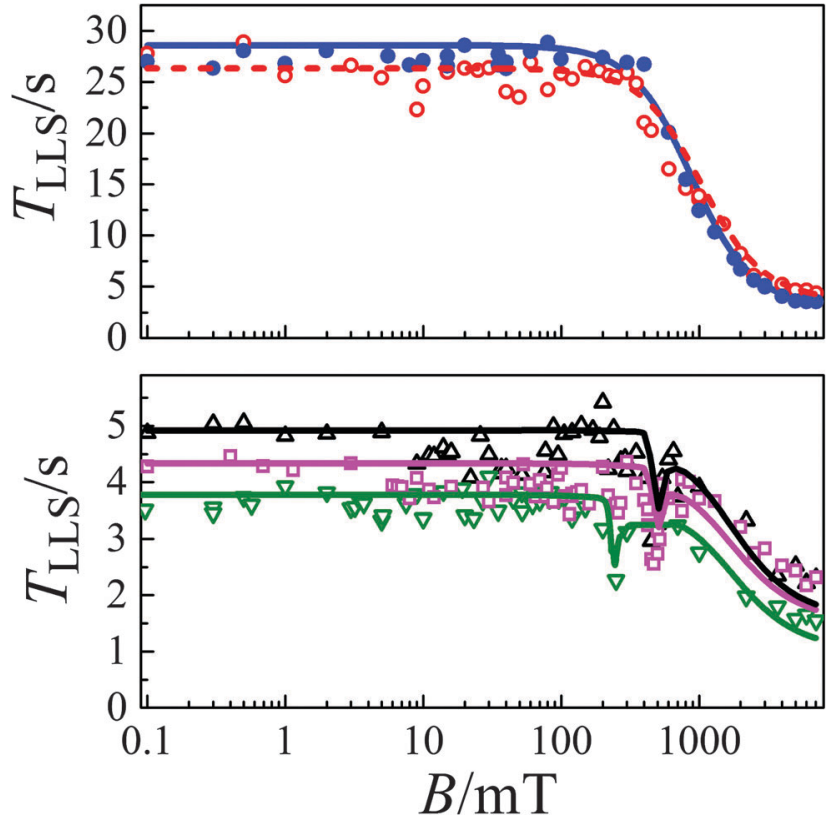

Fig. 3 Field dependencies of $T_{\text {LLS }}$ of the $\beta-\mathrm{CH}_{2}$ protons of $N$-AC-Tyr (down triangles), Tyr (squares), Tyr- $D_{3,5}$ (up triangles), Tyr- $D_{2,6, \alpha}$ (open circles), and Tyr- $D_{2,3,5,6, \alpha}$ (filled circles). $T_{\text {LLS }}$ of the $\beta-\mathrm{CH}_{2}$ protons of Tyr$D_{2,3,5,6, \alpha}$ and $T y r-D_{2,6, \alpha}$ are 45 times longer than the $T_{1}$-relaxation time at $7 \mathrm{~T}$. Solid lines show the calculation results.

In both cases $T_{\mathrm{LLS}}$ at low fields is about $27 \mathrm{~s}$ while $T_{1}$ at $7 \mathrm{~T}$ is about $0.61 \mathrm{~s}$, resulting in an increase of the HP lifetime by a factor of $T_{\mathrm{LLS}} / T_{1}=43$ as compared to the longitudinal spin order. The fact that the difference in $T_{\mathrm{LLS}}$ is small for Tyr$\mathrm{D}_{2,3,5,6, \alpha}$ and Tyr- $\mathrm{D}_{2,6, \alpha}$ is not surprising: remote protons in the $\mathrm{H} 3,5$ positions only slightly affect the relaxation behaviour of the $\beta-\mathrm{CH}_{2}$ protons, an effect that can be accounted for by adjusting the $R_{0}$ value (also there is no sizeable direct scalar coupling between the $\beta-\mathrm{CH}_{2}$ protons and the $\mathrm{H} 3, \mathrm{H} 5$ protons).

Now let us increase the complexity of the spin system and consider the field dependencies of $T_{\mathrm{LLS}}$ in the other three tyrosine derivatives (Fig. 3). We have found that $T_{\mathrm{LLS}}$ at low magnetic field increases in the sequence from $N$-Ac-Tyr $(3.8 \mathrm{~s})$, to Tyr $(4.3 \mathrm{~s})$ and, finally, to Tyr- $\mathrm{D}_{3,5}\left(4.9 \mathrm{~s}\right.$ ) (see Fig. 3) while $T_{1}$ at $7 \mathrm{~T}$ is almost the same, about $0.61 \mathrm{~s}$. In all cases the ratio $T_{\mathrm{LLS}} / T_{1}$ is only about 6-8 due to the influence of the neighbouring protons $(\alpha-\mathrm{CH}, \mathrm{H} 2, \mathrm{H} 6)$. In order to increase $T_{\mathrm{LLS}}$ it is necessary to first substitute the proton nearest to the $\beta-\mathrm{CH}_{2}$ protons (in this case this is the $\alpha-\mathrm{CH}$ proton), by a deuteron. In the field dependence there is again a sharp feature at fields around $250 \mathrm{mT}$ for $N$-Ac-Tyr and around $500 \mathrm{mT}$ for Tyr and Tyr- $\mathrm{D}_{3,5}$. As in the case of $\mathrm{N}$-Ac-His it is due to an LAC in the three-spin system of the $\alpha-\mathrm{CH}$ and $\beta-\mathrm{CH}_{2}$ protons between the same spin states as discussed before. We have found that even when other protons stay far away from the $\beta-\mathrm{CH}_{2}$ protons, like the acetyl group in $\mathrm{N}$-Ac-Tyr or the $\mathrm{H} 3, \mathrm{H} 5$ protons in $N$-Tyr and Tyr, they still contribute to the relaxation of the LLS but do not change the main trend of increasing $T_{\mathrm{LLS}}$ with decreasing magnetic field. Removing the acetyl group leads to an increase of $T_{\mathrm{LLS}}$ by $15 \%$ while further deuteration of the $\mathrm{H} 3$ and $\mathrm{H} 5$ protons gives a total gain of 34\%. Different LAC positions in the three tyrosine compounds are caused by the changes in the chemical shift of the protons: the chemical shift difference between the $\alpha-\mathrm{CH}$ and $\beta-\mathrm{CH}_{2}$ protons increases by a factor of 1.9 in the presence of the acetyl group. As a result, the LAC field decreases by the same factor, as was found experimentally and reproduced by the theoretical model.

The difference between the relaxation dispersion of the LLS with its singlet character and that of other spin orders is rather pronounced. Because of the nature of photo-CIDNP at low magnetic field not only the LLS is formed but other spin orders as well. Their lifetimes have also been measured; in particular we can compare the behaviour of $T_{\mathrm{LLS}}$ and $T_{1}$. As shown above the LLS lifetime increases with decreasing magnetic field, whereas the $T_{1}$-relaxation dispersion behaves in the opposite way for some of the protons.

This is the case, for instance, for $T_{1}$ of the $\alpha-\mathrm{CH}$ proton. The $T_{1}$ dispersion of the $\alpha-\mathrm{CH}$ proton in Tyr is shown in Fig. 4 . The $\alpha-\mathrm{CH}$ proton has scalar couplings with the $\beta-\mathrm{CH}_{2}$ protons, which have a faster rate of $T_{1}$-relaxation. It was shown before ${ }^{56,57}$ that in a multi-spin system in the strong coupling regime the spins tend to relax with a common $T_{1}$, which is given by the 'average' rate of their $T_{1}$-relaxation. Therefore $T_{1}$ of the $\alpha$-CH proton, which is $4.5 \mathrm{~s}$ at high field, becomes shorter because of the interaction with the $\beta-\mathrm{CH}_{2}$ protons that have a $T_{1}$ of only $0.62 \mathrm{~s}$ at high field (but now they have partly singlet order). The sharp feature at $500 \mathrm{mT}$ (see Fig. 4) as we discussed above is caused by the LAC at this field. In all cases the presence of an LAC leads to a decrease of $T_{\mathrm{LLS}}$, which is accompanied by an increase of $T_{1}$ of the $\alpha-\mathrm{CH}$ proton. This is because at the LAC region the two crossing states are mixed, hence they start relaxing with the average rate, which leads to the increase of $T_{1}$ and decrease of $T_{\mathrm{LLS}}$.

\section{LLSs in tyrosine in the presence of TEMPOL}

When LLS applications in Magnetic Resonance Imaging (MRI) in vivo are discussed one faces a problem that biological tissues may contain paramagnetic compounds, which reduce the $T_{\mathrm{LLS}}$ time. Also, LLSs are promising for DNP experiments where HP can be converted to long-lived spin order and stored there. The DNP technique is inherently confronted with the presence of stable radicals in solutions (except for special cases ${ }^{54}$ where radicals are quenched chemically), which decreases both $T_{\mathrm{LLS}}$ and $T_{1}$. Therefore studying the influence of paramagnetic additives on $T_{\mathrm{LLS}}$ is an

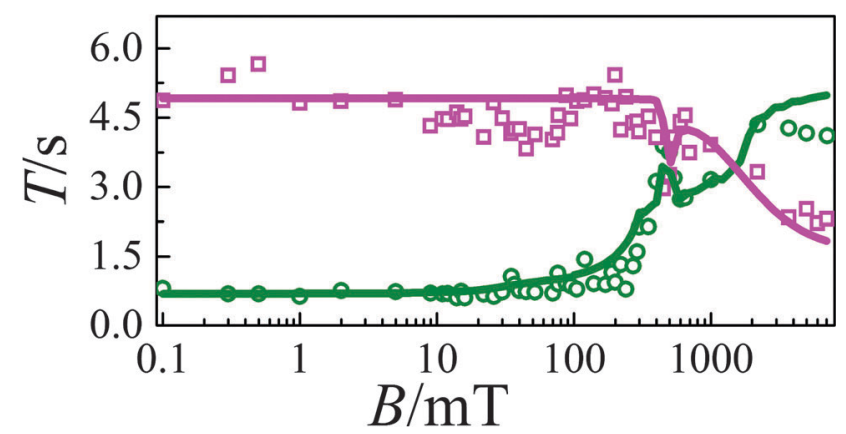

Fig. 4 Field dependencies of $T_{\text {LLS }}$ and $T_{1}$ : $T_{\text {LLS }}$ of the $\beta-\mathrm{CH}_{2}$ protons (squares) and $T_{1}$ of the $\alpha-\mathrm{CH}$ protons (circles) of Tyr. Solid lines show the calculation results. 


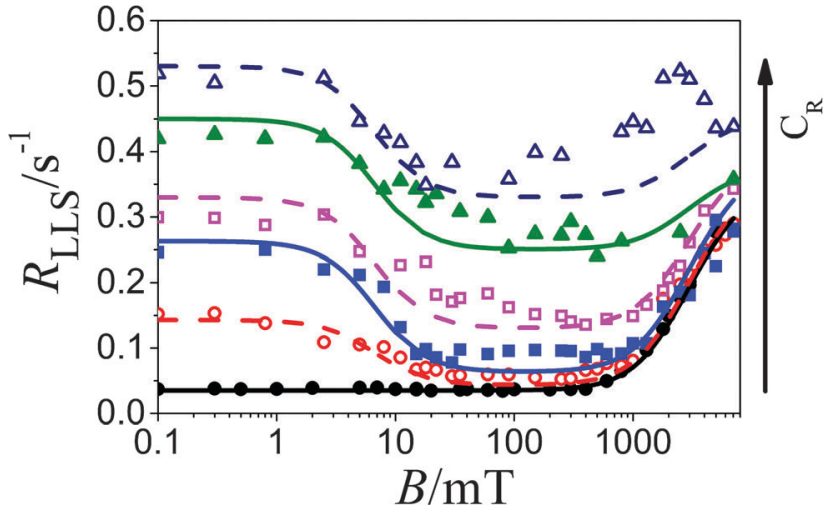

Fig. 5 Field dependencies of $T_{\text {LLS }}$ of the $\beta-\mathrm{CH}_{2}$ protons of $T y r-D_{2,3,5,6, \alpha}$ for different concentrations, $C_{R}$, of added TEMPOL: $0 \mathrm{mM}$ (filled circles), $1 \mathrm{mM}$ (open circles), $1.3 \mathrm{mM}$ (filled squares), $1.6 \mathrm{mM}$ (open squares), $1.75 \mathrm{mM}$ (filled triangles), $2 \mathrm{mM}$ (open triangles). Lines show the calculation results.

important issue for optimizing the performance of such DNP experiments. ${ }^{7}$ In order to investigate the $T_{\mathrm{LLS}}$ field dependencies in the presence of a stable radical the two-spin system of Tyr- $\mathrm{D}_{2,6,3,5, \alpha}$ has been chosen. The nitroxyl radical TEMPOL was used as a paramagnetic agent. The TEMPOL concentration dependence of $R_{\mathrm{LLS}}$ is monotonous (Fig. 5). $T_{\mathrm{LLS}}$ of the sample with $2 \mathrm{mM}$ of TEMPOL and $2 \mathrm{mM}$ Tyr- $\mathrm{D}_{2,6,3,5, \alpha}$ is 4 times longer than $T_{1}$ (without paramagnetic additives $T_{\mathrm{LLS}} / T_{1} \approx 40$ ). This is in qualitative agreement with the results of Tayler and Levitt, ${ }^{7}$ who found that singlet states are less sensitive to the presence of paramagnets than the longitudinal magnetization. This can be accounted for $^{7}$ by partly correlated local fields from paramagnets, which are experienced by the two coupled protons.

To simulate the paramagnetic influence on spin relaxation the following assumptions were used: (i) contributions from different relaxation mechanisms are independent, as a result, the relaxation rate is the sum of the rates; (ii) intramolecular dipolar relaxation does not depend on the TEMPOL concentration; (iii) the $R_{0}$ value coming from additional relaxation mechanisms is fieldindependent. Therefore the following equation for modelling the influence of the paramagnetic agent on $R_{\mathrm{LLS}}$ can be used:

$$
R_{\mathrm{LLS}}\left(B, C_{R}\right)=R_{\mathrm{LLS}}(B)+R_{\mathrm{pm}}\left(B, C_{R}\right)+R_{0}
$$

where $R_{\mathrm{LLS}}(B)$ is the LLS relaxation rate in the absence of TEMPOL (see eqn (1)), $R_{0}$ is a constant that represents additional mechanisms of relaxation, $C_{R}$ is the TEMPOL concentration, $R_{\mathrm{pm}}\left(B, C_{R}\right)$ is the contribution conditioned by the presence of paramagnetic agents, which depends on $C_{R}$ and the external magnetic field. The term $R_{\mathrm{pm}}\left(B, C_{R}\right)$ can be due to either inner-sphere or outer-sphere contributions. ${ }^{58}$ The inner-sphere contribution is coming from molecules bound to the paramagnets; it depends on the field in the following way:

$$
R_{\mathrm{pm}}^{\mathrm{is}}\left(B, C_{R}\right)=\frac{\left(\Delta B\left(C_{R}\right)\right)^{2} \tau_{\mathrm{e}}}{1+\left(\omega(B) \tau_{\mathrm{e}}\right)^{2}}
$$

Here $\Delta B$ is the fluctuating part of the magnetic field near the $\beta-\mathrm{CH}_{2}$ proton caused by the paramagnetic agents, $\omega(B)=\gamma_{\mathrm{H}} B$ is the proton Larmor frequency (with $\gamma_{\mathrm{H}}$ being the proton gyromagnetic ratio), $\tau_{\mathrm{e}}$ is the correlation time of the field fluctuations. Typically, as follows from the BloembergenSolomon-Morgan theory, ${ }^{42}$ the correlation time is of the order of the electron spin relaxation times. The outer-sphere contribution comes from freely diffusing molecules; it is conditioned by translational diffusion, which modulates electron-nuclear dipole-dipole interactions. This contribution can be described by using a model proposed by Hwang and Freed ${ }^{59}$ or more recent models, ${ }^{60-62}$ which give similar results. When the molecule under study and the stable radical do not form a tightly bound complex that rotates as a whole the relaxation is due to the outer-sphere contribution. The $R_{\mathrm{pm}}\left(B, C_{R}\right)$ term is then as follows: ${ }^{63}$

$$
\begin{aligned}
& R_{\mathrm{pm}}^{\mathrm{os}}\left(B, C_{R}\right)=C_{R}\left[\frac{4}{3} J_{\mathrm{d}}\left(\omega_{\mathrm{H}}-\omega_{\mathrm{e}}\right)+4 J_{\mathrm{d}}\left(\omega_{\mathrm{H}}\right)+8 J_{\mathrm{d}}\left(\omega_{\mathrm{H}}+\omega_{\mathrm{e}}\right)\right] \\
& J_{\mathrm{d}}(\omega) \\
& =\frac{15}{2} J_{\mathrm{d}}(0) u^{-5}\left\{u^{2}-2+\mathrm{e}^{-u}\left[\left(u^{2}-2\right) \sin u+\left(u^{2}+4 u+2\right) \cos u\right]\right\}
\end{aligned}
$$

Here $u=\left(\omega \tau_{\mathrm{t}}\right)^{1 / 2}, \tau_{\mathrm{t}}=d^{2} / D$ is the characteristic translational diffusion time ( $d$ is the distance of the closest approach of the molecule and the radial, $D$ is the relative diffusion coefficient); $\omega_{\mathrm{e}}=\gamma_{\mathrm{e}} B\left(\gamma_{\mathrm{e}}\right.$ is the electronic gyromagnetic ratio). The spectral density, $J_{\mathrm{d}}(\omega)$, is conditioned by the modulation of the dipoledipole interaction by relative translational diffusion of the interacting molecules: ${ }^{64}$

$$
J_{\mathrm{d}}(0)=\frac{\pi \gamma_{\mathrm{H}}^{2} \gamma_{\mathrm{e}}^{2} \hbar^{2} \tau_{\mathrm{t}}}{50 d^{3}}
$$

Usually, the outer-sphere mechanism is taken as the main mechanism of nuclear spin relaxation caused by the presence of stable radicals; ${ }^{65}$ however, in some cases considering both contributions becomes necessary. ${ }^{58}$ In our experiments none of the eqn (3) and (4) provides perfect fits of the data; however, eqn (3) better describes the $R_{\mathrm{pm}}\left(B, C_{R}\right)$ contribution. For this reason we used eqn (3) for modelling the data with $(\Delta B)^{2}, R_{0}$ and $\tau_{\mathrm{e}}$ were taken as fitting parameters; we assumed that $\tau_{\mathrm{e}}$ and $R_{0}$ are independent of $C_{R}$; only $(\Delta B)^{2}$ was taken concentrationdependent. The correlation time, $\tau_{\mathrm{e}}$, obtained from fitting was $560 \mathrm{~ns}$, which is similar to the previously measured electron relaxation time. ${ }^{66}$ As is seen from Fig. 5 the simulations are in good agreement with the experimental observation. In this particular case we prefer to show the field dependence of the relaxation rate instead of that of the relaxation time because such a presentation (i) makes the TEMPOL-induced changes better visible and (ii) combination of different mechanisms leads to the summation of the rates, see eqn (2), rather than the times, with only one contribution being $C_{R}$-dependent. Importantly, the $T_{\text {LLS }}$ field dependence is not monotonous anymore in the presence of TEMPOL, but has a maximum at fields around $100 \mathrm{mT}$. The decrease of $T_{\mathrm{LLS}}$ at low magnetic field is caused by the fact that below $10 \mathrm{mT}$ one is in the extreme narrowing regime, which leads to an increase of the relaxation rate $R_{\mathrm{pm}}$ (cf. eqn (3) and (4)). The decrease of $T_{\text {LLS }}$ at high field has 

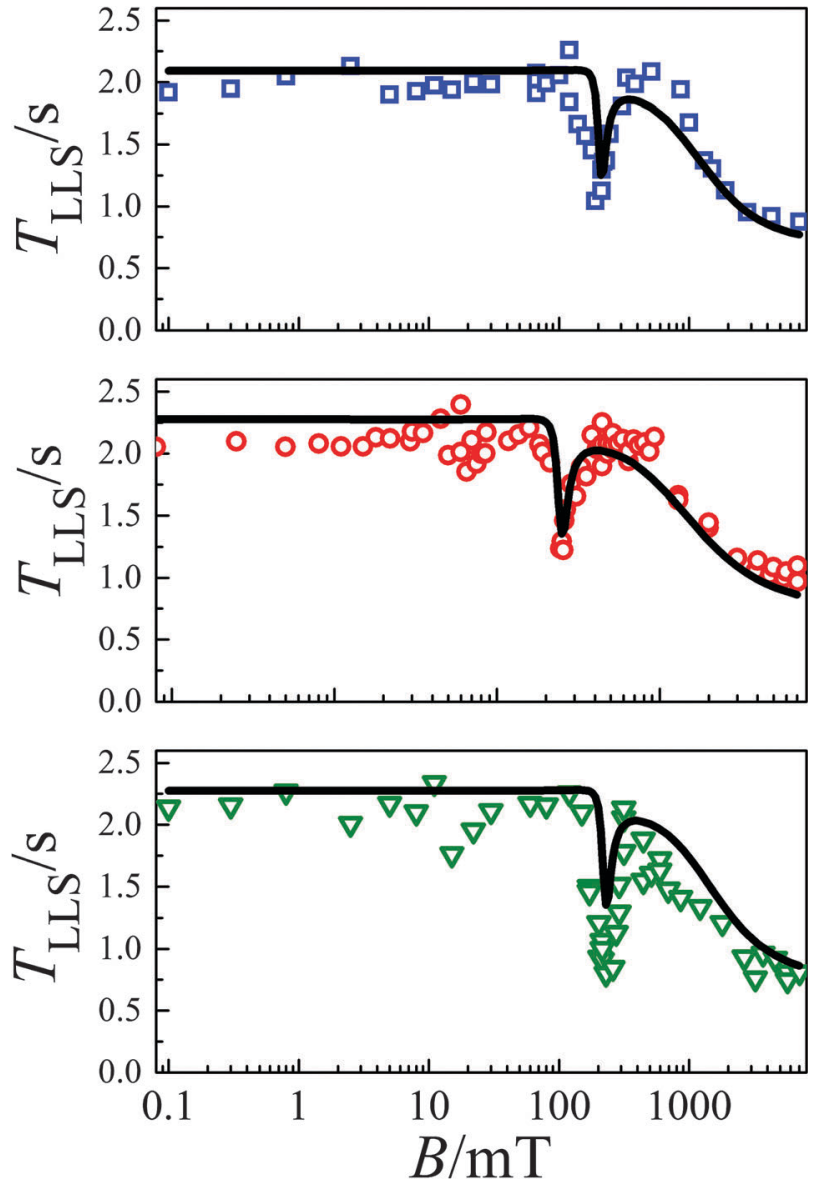

Fig. 6 Field dependencies of $T_{\text {LLS }}$ of the $\beta-\mathrm{CH}_{2}$ protons of the tyrosine residue in two dipeptides: $\mathrm{N}$-Ac-Tyr-Tyr (squares), Trp-Tyr (circles), and in the dyad 4CBP-Tyr (triangles). Correlation time $\tau_{\mathrm{c}}$ was taken $200 \mathrm{ps}$. Solid lines show the calculation results.

already been discussed and is caused by the change in the eigenstates of the static part of the Hamiltonian. This study shows that for samples containing paramagnetic substances the magnetic field value must be carefully optimized, on the one hand low enough to guarantee strong coupling of the nuclear spins so that their singlet state is kept and on the other hand so high that $\omega \tau_{\mathrm{e}} \gg 1$ is fulfilled.

\section{LLSs in tyrosine-containing dyads}

Having investigated the field dependencies of LLSs in free amino acids we continued our study of LLSs by going to larger molecules, namely, dyads, with the aim of assessing longlived spin modes in cases where $T_{1}$-relaxation is so fast that HP can hardly be preserved in the longitudinal spin order. In Tyr-containing dyads $T_{1}$ is about $0.3 \mathrm{~s}$, which is comparable to the times, $\tau_{1}$ and $\tau_{2}$, of field variation thus being at the border of detectable relaxation times in our experiments. For this reason in dyads it becomes crucial to exploit LLSs, which do not relax during the field variation. To compare the data with the previous measurements of $T_{\mathrm{LLS}}$ of the $\beta-\mathrm{CH}_{2}$ protons of the Tyr residue in $N$-Ac-Tyr-Tyr, the dipeptide Tyr-Trp and the dyad 4CBP-Tyr (see Fig. 6) were studied. The $T_{\mathrm{LLS}}$ times of the $\beta-\mathrm{CH}_{2}$ protons in $N$-Ac-Tyr-Tyr at both termini are almost identical therefore in Fig. 6 we show the average $T_{\text {LLS }}$. The three compounds studied have almost the same $T_{1}$ (about $0.3 \mathrm{~s}$ ) and $T_{\text {LLS }}$ (about $2 \mathrm{~s}$ at low field). Hence, increasing the molecular size leads to the decrease of both $T_{\mathrm{LLS}}$ and $T_{1}$ by a factor of 2 , which is attributed to the change in correlation time of the molecular motion from $100 \mathrm{ps}$ to $200 \mathrm{ps}$. Thus, in the fully protonated dyads $T_{\text {LLS }}$ at low field is about 7 times longer than $T_{1}$ at high field, a result that is comparable to the $T_{\mathrm{LLS}} / T_{1}$ ratio in the fully protonated amino acids discussed above. Thus, the long-lived character of HP is preserved in dyads as well. Dips at fields around $200 \mathrm{mT}$ are caused by the LAC in the three-spin systems of the $\alpha-\mathrm{CH}$ and $\beta-\mathrm{CH}_{2}$ protons as it was described above. The slight difference in the LAC field position is caused by the difference in their structure.

\section{Conclusions}

In the present work we systematically studied the relaxation dispersion of long-lived spin states in amino acids with different isotopomers, also in dyads containing a tyrosine residue. We have shown that using photo-CIDNP formation at low magnetic field allowed us to generate an LLS of the $\beta-\mathrm{CH}_{2}$ protons even in multispin systems with up to seven scalar-coupled protons and to study the whole $T_{\text {LLS }}$ field dependence. Using CIDNP gives several advantages: first, HP is generated rapidly and the preparation process is easy to control, while in analogous PHIP and DNP experiments the preparation can take much longer periods of time. Second, the CIDNP technique allows one to overpopulate the LLS without the need of additional NMR pulses and to increase the NMR signal by a factor of $\sim 100$ (although one should note that the NMR enhancement factor in $\mathrm{DNP}^{28,67,68}$ and PHIP ${ }^{18,27,69}$ cases is usually larger). Third, the experiment is carried out at room temperature and does not require cooling the sample down to cryogenic temperatures. Fourth, CIDNP can be formed in various bio-molecules, e.g., amino acids, peptides, and proteins, thus allowing one to increase the lifetime of the $\beta-\mathrm{CH}_{2}$ proton spin order, which has a short $T_{1}$-relaxation time. Typically, with our method one can polarize only amino acids with ring structures, which can be both a difficulty (as other amino acids cannot be assessed with this method) and an advantage (as one can study selected amino acid residues in proteins and peptides). We have shown that in the case of the isolated $\beta-\mathrm{CH}_{2}$ two-spin system an increase of $T_{\mathrm{LLS}}$ by a factor of approximately 45 as compared with $T_{1}$ is achievable. The ratio $T_{\mathrm{LLS}} / T_{1}$ decreases upon increasing the number of protons in the system, down to about 7 in fully protonated amino acids and dyads. We have applied the theory of dipolar relaxation to simulate in all cases studied the relaxation dispersion of the LLS finding good agreement with the experimental observations. This theory also allowed us to explain sharp features in the relaxation dispersion of LLSs caused by LACs. Our experimental and theoretical approach made it possible to measure and model the whole field dependence of $T_{\text {LLS }}$ in multi-spin systems. Previously, studies of LLSs were limited to high fields and very low fields, with only a few exceptions. ${ }^{5,67}$ 
We have also investigated the influence of a paramagnetic agent (stable radical TEMPOL) on the field dependence of $T_{\mathrm{LLS}}$. Our results are in agreement with previous observation of a monotonous increase of $R_{\mathrm{LLS}}$ with an increase of the concentration of the paramagnetic agent. ${ }^{7}$ However, the investigation of whole field dependence allowed us to find out that $T_{\text {LLS }}$ is longest not at the lowest field. We have shown that the longest $T_{\mathrm{LLS}}$ is at fields low enough that protons are strongly coupled, but high enough that the influence from the paramagnetic agent decreases when the motional regime, $\omega \tau_{\mathrm{e}} \gg 1$, is reached. In our experiments, the maximal $T_{\text {LLS }}$ was found at around $100 \mathrm{mT}$. Our study demonstrates that in order to improve the performance of DNP experiments and to store HP in LLSs it is desirable to investigate the whole $T_{\mathrm{LLS}}$ field dependence for the compounds under study. The investigation of the field dependence of $T_{\mathrm{LLS}}$ in amino acids and dyads helps to optimize experiments, in which HP is stored in LLSs, and for improving relaxation studies using LLSs. In addition, from our studies the conclusion can be drawn that the most important step for increasing the LLS lifetimes is substituting the nearest proton being the $\alpha-\mathrm{CH}$ proton, by deuterium: this is by far the easiest position for substitution; moreover, it provides a drastic increase of $T_{\text {LLS }}$. In contrast, other positions are much more difficult to substitute and (when His is taken as an example) provide only an additional two-fold gain in $T_{\mathrm{LLS}} / T_{1}$.

The long $T_{\text {LLS }}$ times that we obtained can be used to study slow dynamic processes by NMR. Notably, one can harness LLSs to study $^{31,32}$ very slow chemical exchange or translational diffusion and protein unfolding. ${ }^{70}$ Recent examples of MRI using long-lived spin order show ${ }^{71,72}$ that LLSs are promising for in vivo and clinical applications. Since delivering hyper-polarized molecules to the observation position takes certain time, during which hyperpolarization relaxes, exploiting LLSs can be promising to sustain signal enhancement in MRI experiments. In this context long LLS lifetimes reported here can be useful. Because of the possibility to create LLSs in amino acids and peptides one can also exploit long $T_{\text {LLS }}$ times to study slow dynamics in biomolecules.

\section{Acknowledgements}

We are thankful to Dr Gerald Hörner (Adam Mickiéwicz University, Poznan, Poland) for providing the 4CBP-Tyr dyad. This work was supported by the Russian Foundation for Basic Research (projects 13-03-00437, 14-03-00397), the Research Group Linkage Program of the Alexander von Humboldt Foundation, EU-COST Action TD1103, the Ministry of Education and Science of the Russian Federation and grant of the President of RF MD-3279.2014.2.

\section{Notes and references}

1 M. Carravetta, O. G. Johannessen and M. H. Levitt, Phys. Rev. Lett., 2004, 92, 153003.

2 M. H. Levitt, in Ann. Rev. Phys. Chem., ed. M. A. Johnson and T. J. Martinez, Annual Reviews, Palo Alto, 2012, vol. 63, pp. 89-105.
3 G. Pileio, Prog. Nucl. Magn. Reson. Spectrosc., 2010, 56, 217-231.

4 A. N. Pravdivtsev, K. L. Ivanov, R. Kaptein and A. V. Yurkovskaya, Appl. Magn. Reson., 2013, 44, 23-39.

5 A. S. Kiryutin, S. E. Korchak, K. L. Ivanov, A. V. Yurkovskaya and H.-M. Vieth, J. Phys. Chem. Lett., 2012, 3, 1814-1819.

6 M. B. Franzoni, L. Buljubasich, H. W. Spiess and K. Münnemann, J. Am. Chem. Soc., 2012, 134, 10393-10396.

7 M. C. D. Tayler and M. H. Levitt, Phys. Chem. Chem. Phys., 2011, 13, 9128-9130.

8 M. C. D. Tayler, S. Marie, A. Ganesan and M. H. Levitt, J. Am. Chem. Soc., 2010, 132, 8225-8227.

9 G. Pileio and M. H. Levitt, J. Chem. Phys., 2009, 130, 214501.

10 P. Ahuja, R. Sarkar, P. R. Vasos and G. Bodenhausen, ChemPhysChem, 2009, 10, 2217-2220.

11 M. Carravetta and M. H. Levitt, J. Chem. Phys., 2005, 122, 214505.

12 M. Carravetta and M. H. Levitt, J. Am. Chem. Soc., 2004, 126, 6228-6229.

13 A. A. Karabanov, C. Bretschneider and W. Köckenberger, J. Chem. Phys., 2009, 131, 204105.

14 G. Pileio, M. Carravetta, E. Hughes and M. H. Levitt, J. Am. Chem. Soc., 2008, 129, 12582-12583.

15 G. Pileio, M. Concistré, M. Carravetta and M. H. Levitt, J. Magn. Reson., 2006, 182, 353-357.

16 G. Pileio and M. H. Levitt, J. Magn. Reson., 2007, 187, 141-145.

17 K. Gopalakrishnan and G. Bodenhausen, J. Magn. Reson., 2006, 182, 254-259.

18 E. Vinogradov and A. K. Grant, J. Magn. Reson., 2008, 194, 46-57.

19 A. K. Grant and E. Vinogradov, J. Magn. Reson., 2008, 193, 177-190.

20 T. Maly, G. T. Debelouchina, V. S. Bajaj, K.-N. Hu, C.-G. Joo, M. L. Mak-Jurkauskas, J. R. Sirigiri, P. C. A. van der Wel, J. Herzfeld, R. J. Temkin and R. G. Griffin, J. Chem. Phys., 2008, 128, 052211.

21 K. H. Hausser and D. Stehlik, Adv. Magn. Reson., 1968, 3, 79.

22 C. Griesinger, M. Bennati, H.-M. Vieth, C. Luchinat, G. Parigi, P. Höfer, F. Engelke, S. J. Glaser, V. Denysenkov and T. F. Prisner, Prog. Nucl. Magn. Reson. Spectrosc., 2012, 64, 4-28.

23 R. A. Green, R. W. Adams, S. B. Duckett, R. E. Mewis, D. C. Williamson and G. G. R. Green, Prog. Nucl. Magn. Reson. Spectrosc., 2012, 67, 1-48.

24 J. Natterer and J. Bargon, Prog. Nucl. Magn. Reson. Spectrosc., 1997, 31, 293-315.

25 K. M. Salikhov, Y. N. Molin, R. Z. Sagdeev and A. L. Buchachenko, Spin Polarization and Magnetic Effects in Chemical Reactions, Elsevier, Amsterdam, 1984.

26 M. B. Franzoni, D. M. Graafen, L. Buljubasich, L. M. Schreiber, H. W. Spiess and K. Münnemann, Phys. Chem. Chem. Phys., 2013, 15, 17233-17239.

27 D. Canet, S. Bouguet-Bonnet, C. Aroulanda and F. Reineri, J. Am. Chem. Soc., 2007, 129, 1445-1449.

28 P. Ahuja, R. Sarkar, S. Jannin, P. R. Vasos and G. Bodenhausen, Chem. Commun., 2010, 46, 8192-8194.

29 G. Pileio, M. Carravetta and M. H. Levitt, Proc. Natl. Acad. Sci. U. S. A., 2010, 107, 17135-17139. 
30 M. C. D. Tayler, I. Marco-Rius, M. I. Kettunen, K. M. Brindle, M. H. Levitt and G. Pileio, J. Am. Chem. Soc., 2012, 134, 7668-7671.

31 R. Sarkar, P. R. Vasos and G. Bodenhausen, J. Am. Chem. Soc., 2007, 129, 328-334.

32 R. Sarkar, P. Ahuja, D. Moskau, P. R. Vasos and G. Bodenhausen, ChemPhysChem, 2007, 8, 2652-2656.

33 R. Kaptein and J. A. den Hollander, J. Am. Chem. Soc., 1972, 94, 6269-6280.

34 V. F. Tarasov and I. A. Shkrob, J. Magn. Reson., Ser. A, 1994, 109, 65-73.

35 K. L. Ivanov, H.-M. Vieth, K. Miesel, A. V. Yurkovskaya and R. Z. Sagdeev, Phys. Chem. Chem. Phys., 2003, 5, 3470-3480.

36 S. Grosse, F. Gubaydullin, H. Scheelken, H.-M. Vieth and A. V. Yurkovskaya, Appl. Magn. Reson., 1999, 17, 211-225.

37 K. L. Ivanov, N. N. Lukzen, H.-M. Vieth, S. Grosse, A. V. Yurkovskaya and R. Z. Sagdeev, Mol. Phys., 2002, 100, 1197-1208.

38 A. B. Doktorov and P. A. Purtov, Sov. Chem. Phys., 1987, 6, 484.

39 P. A. Purtov and A. B. Doktorov, Chem. Phys., 1993, 178, 47-65. 40 A. N. Pravdivtsev, A. V. Yurkovskaya, R. Kaptein, K. Miesel, H.-M. Vieth and K. L. Ivanov, Phys. Chem. Chem. Phys., 2013, 15, 14660-14669.

41 A. N. Pravdivtsev, A. V. Yurkovskaya, H.-M. Vieth and K. L. Ivanov, J. Chem. Phys., 2013, 139, 244201.

42 J. Kowalewski and L. Mäler, in Nuclear spin relaxation in liquids: Theory, experiments, and applications, ed. H. J. Moore and N. D. Spencer, Chem. Phys., CRC Press, Boca Raton, FL., 2006.

43 M. G. Pravica and D. P. Weitekamp, Chem. Phys. Lett., 1988, 145, 255-258.

44 O. B. Morozova, K. L. Ivanov, A. S. Kiryutin, R. Z. Sagdeev, T. Köchling, H.-M. Vieth and A. V. Yurkovskaya, Phys. Chem. Chem. Phys., 2011, 13, 6619-6627.

45 F. Neese, Wiley Interdiscip. Rev.: Comput. Mol. Sci., 2012, 2, 73-78.

46 G. Hörner, G. L. Hug, A. Lewandowska, F. Kazmierczak and B. Marciniak, Chem.-Eur. J., 2009, 15, 3061-3064.

47 G. Hörner, G. L. Hug, D. Pogocki, P. Filipiak, W. Bauer, A. Grohmann, A. Lämmermann, T. Pedzinski and B. Marciniak, Chem.-Eur. J., 2008, 14, 7913-7929.

48 E. G. Rozantsev, Free Nitroxide Radicals, Plenum Press, New York, London, 1970.

49 D. V. Griffits, J. Feeney, G. C. K. Roberts and A. S. V. Burgen, Biochim. Biophys. Acta, 1976, 446, 479-485.

50 I. Schlichting, J. John, M. Frech, P. Chardin, A. Wittinghofer, H. Zimmermann and P. Rösch, Biochemistry, 1990, 29, 504-511.

51 Y. P. Tsentalovich, O. B. Morozova, A. V. Yurkovskaya, P. J. Hore and R. Z. Sagdeev, J. Phys. Chem. A, 2000, 104, 6912-6916.
52 Y. P. Tsentalovich, O. B. Morozova, A. V. Yurkovskaya and P. J. Hore, J. Phys. Chem. A, 1999, 103, 5362-5368.

53 O. B. Morozova, R. Kaptein and A. V. Yurkovskaya, J. Phys. Chem. B, 2012, 116, 12221-12226.

54 P. Miéville, P. Ahuja, R. Sarkar, S. Jannin, P. R. Vasos, S. Gerber-Lemaire, M. Mishkovsky, A. Comment, R. Gruetter, O. Ouari, P. Tordo and G. Bodenhausen, Angew. Chem., Int. Ed., 2010, 49, 6182-6185.

55 K. Miesel, K. L. Ivanov, A. V. Yurkovskaya and H.-M. Vieth, Chem. Phys. Lett., 2006, 425, 71-76.

56 S. E. Korchak, K. L. Ivanov, A. V. Yurkovskaya and H.-M. Vieth, J. Chem. Phys., 2010, 133, 194502.

57 K. L. Ivanov, A. V. Yurkovskaya and H.-M. Vieth, J. Chem. Phys., 2008, 129, 234513.

58 M. Bennati, C. Luchinat, G. Parigi and M.-T. Türke, Phys. Chem. Chem. Phys., 2010, 12, 5902-5910.

59 L.-P. Hwang and J. H. Freed, J. Chem. Phys., 1975, 63, 4017-4025.

60 Y. Ayant, E. Belorizky, P. H. Fries and J. Rosset, J. Phys. Chem. A, 1977, 38, 325-337.

61 C. F. Polnaszek and R. G. Bryant, J. Chem. Phys., 1984, 81, 4038-4045.

62 H. F. Bennett, R. D. Brown III, S. H. Koenig and H. M. Swartz, Magn. Reson. Med., 1987, 4, 93-111.

63 B. Borah and R. G. Bryant, J. Chem. Phys., 1981, 75, 3297-3300.

64 P. S. Hubbard, Proc. R. Soc. London, Ser. A, 1966, 291, 537-555.

65 P. Miéville, S. Jannin and G. Bodenhausen, J. Magn. Reson., 2011, 201, 137-140.

66 I. Panagiotelis, I. Nicholson and J. M. S. Hutchison, J. Magn. Reson., 2001, 149, 74-84.

67 A. Bornet, S. Jannin and G. Bodenhausen, Chem. Phys. Lett., 2011, 512, 151-154.

68 P. R. Vasos, A. Comment, R. Sarkar, P. Ahuja, S. Jannin, J.-P. Ansermet, J. A. Konter, P. Hautle, B. van den Brandt and G. Bodenhausen, Proc. Natl. Acad. Sci. U. S. A., 2009, 106, 18469-18473.

69 T. Jonischkeit, U. Bommerich, J. Stadler, K. Woelk, H. G. Niessen and J. Bargon, J. Chem. Phys., 2006, 124, 201109.

70 A. Bornet, P. Ahuja, R. Sarkar, L. Fernandes, S. Hadji, S. Y. Lee, A. Haririnia, D. Fushman, G. Bodenhausen and P. R. Vasos, ChemPhysChem, 2011, 12, 2729-2734.

71 G. Pileio, S. Bowen, C. Laustsen, M. C. D. Tayler, J. T. Hill-Cousins, L. J. Brown, R. C. D. Brown, J. H. ArdenkjaerLarsen and M. H. Levitt, J. Am. Chem. Soc., 2013, 135, 5084-5088.

72 C. Laustsen, G. Pileio, M. C. D. Tayler, L. J. Brown, R. C. D. Brown, M. H. Levitt and J. H. Ardenkjaer-Larsen, Magn. Reson. Med., 2013, 68, 1262-1265. 